\title{
On resolving the dark LMA solution at neutrino oscillation experiments
}

\author{
Sandhya Choubey ${ }^{a}$ and Dipyaman Pramanik ${ }^{b}$ \\ ${ }^{a}$ Department of Physics, School of Engineering Sciences, \\ KTH Royal Institute of Technology, AlbaNova University Center, \\ Roslagstullsbacken 21, SE-106 91, Stockholm, Sweden \\ ${ }^{b}$ Theoretical Physics Division, Physical Research Laboratory, \\ Navrangpura, Ahmedabad 380009, India \\ E-mail: choubey@kth.se, dipyaman@prl.res.in
}

ABSTRACT: In presence of non standard interactions (NSI), the solar neutrino data is consistent with two solutions, one close to the standard LMA solution with $\sin ^{2} \theta_{12} \simeq 0.31$ and another with $\sin ^{2} \theta_{12}^{D} \simeq 0.69\left(=1-\sin ^{2} \theta_{12}\right)$. The latter has been called the Dark LMA (DLMA) solution in the literature and essentially brings an octant degeneracy in the measurement of the mixing angle $\theta_{12}$. This $\theta_{12}$ octant degeneracy is hard to resolve via oscillations because of the existence of the so-called "generalised mass hierarchy degeneracy" of the neutrino mass matrix in presence of NSI. One might think that if the mass hierarchy is independently determined in a non-oscillation experiment such as neutrino-less double beta decay, one might be able to break the $\theta_{12}$ octant degeneracy. In this paper we study this in detail in the context of long-baseline experiments $\left(P_{\mu \mu}\right.$ channel $)$ as well as reactor experiments $\left(P_{e e}\right.$ channel) and show that if we combine information from both long-baseline and reactor experiments we can find the correct octant and hence value of $\theta_{12}$. We elaborate the reasons for it and study the prospects of determining the $\theta_{12}$ octant using T2HK, DUNE and JUNO experiments. Of course, one would need information on the neutrino mass hierarchy as well.

KeYwords: Neutrino Detectors and Telescopes (experiments), Oscillation

ArXiv ePrint: 1912.08629 


\section{Contents}

1 Introduction 1

2 Experimental and numerical details 3

2.1 DUNE 3

2.2 T2HK 4

2.3 Simulation techniques 4

3 LMA vs. DLMA and oscillation probabilities 5

4 Mass squared difference and $\theta_{12} \quad 8$

5 Comparing T2HK with DUNE 11

6 Role of neutrino mass hierarchy 12

$\begin{array}{lll}7 & \text { Conclusions } & 13\end{array}$

\section{Introduction}

Within the standard three-generation neutrino oscillation paradigm, the Large Mixing Angle (LMA) solution provides an excellent explanation to the solar neutrino problem [1] and has been independently tested and confirmed by the KamLAND [2] reactor antineutrino experiment. The solar and KamLAND data jointly restrict the LMA solution to $\Delta m_{21}^{2} \simeq 7.5 \times 10^{-5} \mathrm{eV}^{2}$ and $\sin ^{2} \theta_{12} \simeq 0.31$ [3]. In particular, the high significance compatibility of the solar neutrino data with resonant MSW matter effect inside the sun rules out all values of $\sin ^{2} \theta_{12}>0.5$ for $\Delta m_{21}^{2}>0$. However, this apparently robust conclusion runs into trouble $[4,5]$ as soon as one allows for the presence of (large) Non-Standard neutrino Interactions (NSIs) [6]. It has been shown that in presence of NSI we get two nearly degenerate solutions that fits the solar neutrino data - the standard LMA solution $\left(\Delta m_{21}^{2} \simeq 7.5 \times 10^{-5} \mathrm{eV}^{2}, \sin ^{2} \theta_{12} \simeq 0.3\right)$ mentioned above, and another solution at $\left(\Delta m_{21}^{2 D} \simeq 7.5 \times 10^{-5} \mathrm{eV}^{2}, \sin ^{2} \theta_{12}^{D} \simeq 0.7\right)$. This latter solution has been called the Dark LMA (DLMA) solution [4] which has the same best-fit $\Delta m_{21}^{2}$ as in LMA solution but the best-fit mixing angle is $\sin ^{2} \theta_{12}^{D} \simeq 1-\sin ^{2} \theta_{12}$. This is therefore like an octant degeneracy in $\theta_{12}$. The conditions that need to be satisfied for the degeneracy to be exact, include NSIs corresponding to scattering off both up and down type quarks with NSI couplings proportional to their respective charges [7-10]. However even without this assumption, global analysis of solar neutrino and KamLAND data give the above mentioned approximately degenerate solutions at LMA and DLMA [11]. 
The existence of the DLMA solution not only has consequences for model building but has phenomenological implications as well. In particular, once both "octants" of $\theta_{12}$ become viable, neutrino mass hierarchy determination at oscillation experiments becomes a problem owing to the "generalised mass hierarchy degeneracy" [9], which can be understood as follows. For pure vacuum oscillations, the neutrino mass matrix and hence the neutrino oscillation probabilities remain invariant under the simultaneous transformations $\Delta m_{31}^{2} \leftrightarrow-\Delta m_{32}^{2}, \sin ^{2} \theta_{12} \leftrightarrow \cos ^{2} \theta_{12}$ and $\delta_{c p} \leftrightarrow \pi-\delta_{c p}$. This poses a serious challenge for experiments such as JUNO $[12,13]$ that are attempting to measure the sign of $\Delta m_{31}^{2}$ via vacuum oscillations [8]. Presence of matter effects could in principle break this degeneracy, however, if one adds the possibility of having NSI in the neutrino sector, then in addition to the transformations mentioned above if we also transform the NSI parameters $\epsilon_{e e} \rightarrow \epsilon_{e e}-2$, $\epsilon_{e \mu} \rightarrow \epsilon_{e \mu}^{*}, \epsilon_{e \tau} \rightarrow \epsilon_{e \tau}^{*}$ and all other $\epsilon_{\alpha \beta} \rightarrow-\epsilon_{\alpha \beta}^{*}$, then the mass hierarchy degeneracy is restored [9] and now even experiments such as DUNE [14] that aim to determine the sign of $\Delta m_{31}^{2}$ via observation of earth matter effects are unable to do so [9]. As long as this degeneracy remains unresolved, oscillation experiments will not be able to measure the sign of $\Delta m_{31}^{2}$, unless the issue of the octant of $\theta_{12}$ is resolved. Likewise, the octant of $\theta_{12}$ can be determined at oscillation experiments only if the sign of $\Delta m_{31}^{2}$ is known.

Therefore, it is obvious that both cannot be determined simultaneously using oscillation experiments alone and one needs to look for inputs from non-oscillation experiments for the resolution of the LMA vs DLMA conundrum. This can be done in two ways. The first way is of course to independently constrain the NSI parameters themselves such that the values of the NSI parameters required for the DLMA solution are disfavored, and hence disfavoring the DLMA solution $[10,15]$. Constraints on NSI parameters have been obtained from neutrino scattering experiments. However, these constraints depend on the mass of the mediator particle. For heavy mediators, the best constraints come from the CHARM [16] and NuTeV experiments [17], while the current data from COHERENT [18] severely constrains the NSI parameters to mediator masses down to $10 \mathrm{MeV}$. However, there still remains allowed a narrow window of NSI parameters for which the DLMA solution is allowed [19]. Indeed the global analysis [20] of neutrino oscillation data and COHERENT data allows the DLMA solution for certain (restricted) class of NSI models, with some cases even favoring the DLMA solution over the LMA solution (cf. figure 8 of [20]). Future data from CONUS and more data from COHERENT could narrow down this range severely [19], however, this remains to be seen, and it is possible that the DLMA solution could survive the constraints from neutrino scattering experiments for very low mediator masses and/or specific NSI models. Hence, neutrino scattering data may or may not be able to completely rule out the DLMA solution in a truly model independent way.

The other way of resolving the $\theta_{12}$ vs $\theta_{12}^{D}$ conundrum could be to determine the neutrino mass hierarchy in a non-oscillation experiment and then test octant of $\theta_{12}$ in an oscillation experiment. Here, we will study in detail the possibility of determining the octant of $\theta_{12}$ at long-baseline experiments such as T2HK [25] or DUNE [14] and show that owing to an intrinsic correlation between $\theta_{12}$ and $\left|\Delta m_{31}^{2}\right|$ in $P_{\mu \mu}$, it is impossible to say anything about $\theta_{12}$ at all from data from long-baseline experiments alone, even if the sign of $\Delta m_{31}^{2}$ was known. We will then show how we can break this correlation using data from a complementary oscillation experiment such as JUNO which measures $P_{e e}$. We show that 
while $\Delta m_{31}^{2}$ and $\sin ^{2} \theta_{12}$ are correlated for $P_{e e}$, they are anti-correlated for $P_{\mu \mu}$. Therefore, adding information from both channels can break the $\theta_{12}$ octant degeneracy.

Some comments on our assumption that neutrino mass hierarchy will be determined in a non-oscillation experiment is in order. The main non-oscillation experiments which depend on the neutrino mass hierarchy are neutrino-less double beta decay experiments and cosmology. The implications of DLMA for neutrino-less double beta decay have been presented in [21]. It was pointed out in that work that predictions for neutrino-less double beta decay for inverted hierarchy $(\mathrm{IH})$ remain invariant under change from LMA to DLMA. However, for normal hierarchy $(\mathrm{NH})$, the predicted value of the effective mass $m_{\beta \beta}$ shifts to higher values into the "desert region", increasing the prospects of a positive signal in this very important class of experiments. Since the next generation neutrino-less double beta decay experiments are expected to cover the entire predicted range for $\mathrm{IH}$, we could know the neutrino mass hierarchy in the near future, if neutrinos are Majorana particles. While this is an optimistic view-point, one must keep in mind the caveats associated with it. Firstly, as pointed out above, non-observation of neutrino-less double beta decay does not necessarily imply that IH is ruled out - it may well be that the neutrinos are not Majorana particles. On the other hand, if we do observe a positive signal, this would clearly prove that neutrinos are Majorana. However, this would still not be fool-proof confirmation of IH since a variety of new physics scenarios (see for e.g. [22]) could lead to a neutrinoless double beta half-life that is comparable to that predicted by neutrino masses with IH. Separation between the predicted $m_{\beta \beta}$ for $\mathrm{NH}$ and $\mathrm{IH}$ also depends on the lightest neutrino mass, and the limits on this are obtained from cosmological data, which have their own set of caveats. Finally, $m_{\beta \beta}$ depends on the nuclear matrix element uncertainties and this needs to be taken into consideration when drawing conclusions from neutrino-less double beta decay experiments. Determining the neutrino mass hierarchy to some extent is also possible from future cosmological data (see e.g. [23]).

The rest of the paper is organised as follows. In section 2, we give a brief description of our numerical techniques and experimental details. In section 3 we discuss the neutrino oscillation probabilities to show how the long-baseline experiments and reactor experiments depend on the octant of $\theta_{12}$. In section 4 we discuss the (anti)correlation between $\theta_{12}$ and $\left|\Delta m_{31}^{2}\right|$ and the role of combining JUNO with T2HK (or DUNE) in breaking this correlation, thereby solving the $\theta_{12}$ octant degenracy. In section 5 we compare the sensitivity of T2HK vs DUNE in resolving the DLMA solution. Section 6 discusses the role of neutrino-less double beta decay experiments briefly. Finally, we end in section 7 with our conclusions.

\section{Experimental and numerical details}

We first describe here the specifications we use in our analysis for the DUNE and T2HK experiments. We next detail the simulation technique used in our analysis.

\section{$2.1 \quad$ DUNE}

Deep Underground Neutrino Experiment (DUNE) is a future long-baseline experiment program to be built in the U.S.A. . It will have an intense neutrino source at Fermilab 
and a far detector $1300 \mathrm{~km}$ away at the Sanford Underground Research Facility in South Dakota. The neutrino beam will be generated by a $80-120 \mathrm{GeV}$ proton beam at $1.2-2.4$ MW. The far detector will be made of 4 identical $10 \mathrm{kt}$ liquid Argon detectors which will give a fiducial mass of $34 \mathrm{kt}$.

In this work we followed the specifications given in [24]. We have considered a 34 kt. detector at baseline $1300 \mathrm{~km}$. The neutrino flux is given by the $120 \mathrm{GeV}, 1.2 \mathrm{MW}$ proton beam. Liquid Argon detectors can measure both $\mu$ and $e$ type events. So we have taken into consideration both the $\nu_{e}\left(\bar{\nu}_{e}\right)$ appearance as well as the $\nu_{\mu}\left(\bar{\nu}_{\mu}\right)$ disappearance channels in the analysis. We consider 5 years running of the experiment in the neutrino mode and 5 years in the antineutrino mode. We have taken $20 \% / \sqrt{E}$ energy resolution for the $\mu$ and $15 \% / \sqrt{E}$ resolution for the $e$ channels. We took the signal efficiency to be $85 \%$. For the neutrino (antineutrino) mode, the signal normalization error is taken as $2 \%(5 \%)$, the background normalization error is taken as 10\%(10\%) and the energy calibration error is taken as $5 \%(5 \%)$. Although there is an updated version of the DUNE specifications available in the literature [14], we explicitly checked that the main conclusions of our analysis do not change significantly for the new specifications. So we have kept the older specifications in our analysis.

\subsection{T2HK}

T2HK [25] is the upgradation program of the currently running T2K experiment [26]. Here the neutrino beam will come from an upgraded J-PARC accelerator source at Tokai. The far detector will be an upgraded version of the currently running Super-Kamiokande (SK) detector, and will be called Hyper-Kamiokande (HK). HK will be made of two identical $187 \mathrm{kt}$ water Cherenkov detectors which will be placed at a baseline of about $295 \mathrm{~km}$ from the source. The HK detector will be at a location $2.5^{\circ}$ off-axis with respect to the J-PARC beam-line.

For this work we consider $1.3 \mathrm{MW}$ beam power and the $2.5^{\circ}$ off-axis flux. We take $295 \mathrm{~km}$ as the baseline and $374 \mathrm{kt}$ as the fiducial mass. For the run-time we consider 2.5 years running in the neutrino mode and 7.5 years running in the antineutrino mode. We match our simulated number of events with tables 2 and 3 of [27]. We take $15 \% / \sqrt{E}$ energy resolution for all channels. The signal normalization errors for $\nu_{e}\left(\bar{\nu}_{e}\right)$ and $\nu_{\mu}\left(\bar{\nu}_{\mu}\right)$ channel are $3.2 \%(3.6 \%)$ and $3.9 \%(3.6 \%)$, respectively. We also take $10 \%$ and $5 \%$ background and energy calibration errors in all channels, respectively.

We stress that we have not done a full simulation of the JUNO experiment. Instead we just put the expected constraints obtained by the JUNO collaboration [12] as a prior in our analysis. While we understand that a full analysis would of course be more desirable, however, since the purpose of this paper is only to present the idea that one can use to find the octant of $\theta_{12}$, this serves the purpose. For a more rigorous quantitative analysis, one could simulate the JUNO data. We leave that for a future work.

\subsection{Simulation techniques}

We have used GLoBES (Global Long-baseline Experiment Simulator) [28, 29] for our simulation of the long-baseline experiments. For the calculation of neutrino oscillation prob- 


\begin{tabular}{|l|l|l|}
\hline Parameter Name & True value & Marginalization \\
\hline $\sin ^{2} \theta_{12}$ & $0.31(0.69)$ & {$[0.0-1.0]$} \\
\hline$\theta_{13}$ & $8.48^{\circ}$ & Nil \\
\hline$\theta_{23}$ & $42^{\circ}$ & Nil \\
\hline$\delta_{\mathrm{CP}}$ & $-90^{\circ}$ & {$[-\pi, \pi]$} \\
\hline$\Delta m_{21}^{2}$ & $7.50 \times 10^{-5} \mathrm{eV}^{2}$ & $\mathrm{Nil}$ \\
\hline$\Delta m_{31}^{2}$ & $2.52 \times 10^{-3} \mathrm{eV}^{2}$ & {$[2.3,2.6] \times 10^{-3} \mathrm{eV}^{2}$} \\
\hline$\epsilon_{e e}$ & 0 & {$[-3.0,3.0]$} \\
\hline$\epsilon_{\alpha \beta}, \alpha \beta \neq e e$ & 0 & $\mathrm{Nil}$ \\
\hline
\end{tabular}

Table 1. Values of parameters taken for generating mock data used in this work and the range over which they are marginalised in the fit.

abilities in presence of NSI, we used NSI probability engine of MonteCUBES [30]. We have taken the following values of the oscillation parameters for generating our mock data: $\sin ^{2} \theta_{12}=0.31, \theta_{13}=8.48^{\circ}, \theta_{23}=42^{\circ}, \delta_{\mathrm{CP}}=-90^{\circ}, \Delta m_{21}^{2}=7.5 \times 10^{-5} \mathrm{eV}^{2}$ and $\Delta m_{31}^{2}=2.52 \times 10^{-3} \mathrm{eV}^{2}$. This is consistent with the currently allowed values of the standard oscillation parameters from global fits [3]. We assume normal hierarchy in data for all the results presented in the paper, except the last figure, where inverted hierarchy is taken in the mock data. In the fit we marginalise over standard oscillation parameters $\Delta m_{31}^{2}$ and $\delta_{\mathrm{CP}}$. The range used in our maginalization is given in the table 1 and is consistent with the currently allowed ranges. Note that for $\theta_{12}$ we consider the entire range in the fit since studying the octant degeneracy of $\theta_{12}$ is the main concern of this work. We keep $\Delta m_{21}^{2}$ fixed in the fit since varying it has practically no impact on the final conclusions. We keep $\theta_{23}$ fixed because we explicitly checked that the effect of $\theta_{23}$ marginalisation on the final results is negligible. We also keep $\theta_{13}$ fixed in the fit since it is well determined by the Daya Bay data. While mass hierarchy is considered fixed in most of the plots since we assume normal hierarchy throughout this work, for figure 5 we marginalise over the mass hierarchy in order to show the impact of the generalised mass hierarchy degeneracy and the importance of measuring the mass hierarchy in a non-oscillation experiment.

The mock data is always generated for no NSIs, since the data always correspond to the LMA solution. Whenever we allow NSI to vary in the fit we marginalised over a large range of $\epsilon_{e e}$. We keep all other NSI parameters at zero in both data and the fit. While this choice of marginalisation is not the most general one, it is sufficient for the purpose on this paper. The choices of all the parameters in the data and the marginalization ranges in the fit are summarized in table. 1.

\section{LMA vs. DLMA and oscillation probabilities}

All results in this work are obtained within the exact three-generation paradigm, taking earth matter effect into account. However, the resolution of the octant of $\theta_{12}$ can be understood simply from the expressions of oscillation probabilities in vacuum. Therefore for simplicity, we begin by discussing oscillation probabilities in vacuum. In vacuum, the 
$\nu_{\mu}$ survival probability using the standard parametrisation of the neutrino mixing matrix is given as

$$
\begin{aligned}
P_{\mu \mu}= & 1-P_{21} \sin ^{2}\left(\frac{\Delta m_{21}^{2} L}{4 E}\right) \\
& -4 s_{23}^{2} c_{13}^{2}\left(c_{12}^{2} c_{23}^{2}+s_{12}^{2} s_{23}^{2} s_{13}^{2}-\frac{1}{2} \sin 2 \theta_{12} \sin 2 \theta_{23} s_{13}^{2} \cos \delta\right) \sin ^{2}\left(\frac{\Delta m_{31}^{2} L}{4 E}\right) \\
& -4 s_{23}^{2} c_{13}^{2}\left(s_{12}^{2} c_{23}^{2}+c_{12}^{2} s_{23}^{2} s_{13}^{2}-\frac{1}{2} \sin 2 \theta_{12} \sin 2 \theta_{23} s_{13}^{2} \cos \delta\right) \sin ^{2}\left(\frac{\Delta m_{32}^{2} L}{4 E}\right) .
\end{aligned}
$$

Here, $P_{21}$ is given by

$$
\begin{aligned}
P_{21}= & \sin ^{2} 2 \theta_{12}\left(c_{23}^{4}+s_{23}^{4} s_{13}^{2}\right)+\left(1-\frac{1}{2} \sin ^{2} 2 \theta_{12}\right) \sin ^{2} 2 \theta_{23} s_{13}^{2} \\
& +\sin 4 \theta_{12} \sin 2 \theta_{23}\left(c_{23}^{2}-s_{23}^{2} s_{13}^{2}\right) s_{13} \cos \delta_{13}-\sin ^{2} 2 \theta_{12} \sin ^{2} 2 \theta_{23} s_{13}^{2} \cos ^{2} \delta_{13} .
\end{aligned}
$$

where $\Delta m_{i j}^{2}=m_{i}^{2}-m_{j}^{2}, s_{i j}^{2}$ and $c_{i j}^{2}$ imply $\sin ^{2} \theta_{i j}$ and $\cos ^{2} \theta_{i j}$, respectively, while $E$ and $L$ are the neutrino energy and distance travelled. $P_{21}$ associated with the $\Delta m_{21}^{2}$ driven oscillatory term is symmetric with respect to the interchange $\sin ^{2} \theta_{12} \leftrightarrow \cos ^{2} \theta_{12}$ and hence not interesting to us. The expression in eq. (3.1) shows that $P_{\mu \mu}$ is invariant under simultaneous exchange of $\sin ^{2} \theta_{12} \leftrightarrow \cos ^{2} \theta_{12}$ and $\left|\Delta m_{31}^{2}\right| \leftrightarrow\left|\Delta m_{32}^{2}\right|$. Therefore, if the goal is to distinguish $\sin ^{2} \theta_{12}$ from $\cos ^{2} \theta_{12}\left(=\sin ^{2} \theta_{12}^{D}\right)$, the experiment should be able to distinguish the oscillatory frequency corresponding to $\left|\Delta m_{31}^{2}\right|$ from the frequency corresponding to $\left|\Delta m_{32}^{2}\right|$. The difference in $P_{\mu \mu}$ between $\sin ^{2} \theta_{12}$ and $\sin ^{2} \theta_{12}^{D}=\cos ^{2} \theta_{12}$ is given as,

$$
\Delta P_{\mu \mu}=4 s_{23}^{2} c_{13}^{2} \cos 2 \theta_{12}\left(c_{23}^{2}-s_{23}^{2} s_{13}^{2}\right) \sin \left(\frac{\Delta m_{21}^{2} L}{4 E}\right) \sin \left(\frac{\left(2 \Delta m_{31}^{2}-\Delta m_{21}^{2}\right) L}{4 E}\right) .
$$

Note that this difference goes to zero close to the oscillation maximum corresponding to $\Delta m_{31}^{2}$, and is extremal when $\sin \left(2 \Delta m_{31}^{2}-\Delta m_{21}^{2}\right) L / 4 E \simeq 1$. The difference will also go to zero if $c_{23}^{2}=s_{23}^{2} s_{13}^{2}$, which is safely avoided for the current allowed values of $\theta_{13}$ and $\theta_{23}$. The corresponding difference in the $P_{\mu e}$ channel between $\sin ^{2} \theta_{12}$ and $\sin ^{2} \theta_{12}^{D}$ is

$$
\Delta P_{\mu e}=s_{23}^{2} \sin ^{2} 2 \theta_{13} \cos 2 \theta_{12} \sin \left(\frac{\Delta m_{21}^{2} L}{4 E}\right) \sin \left(\frac{\left(2 \Delta m_{31}^{2}-\Delta m_{21}^{2}\right) L}{4 E}\right) .
$$

In figure 1 we show $\Delta P_{\mu \mu}$ and $\Delta P_{\mu e}$ as a function of energy for the T2HK baseline of $L=295 \mathrm{~km}$ for normal $(\mathrm{NH})$ and inverted $(\mathrm{IH})$ mass hierarchy. We define $\mathrm{NH}$ and $\mathrm{IH}$ corresponding to $\Delta m_{31}^{2}>0$ and $\Delta m_{31}^{2}<0$, respectively. For a given mass hierarchy, extrema in $\Delta P_{\mu \mu}$ and $\Delta P_{e \mu}$ appear close to $E \sim \Delta m_{31}^{2} L /(2 n+1) \pi$, with $n=2,1,0$. We see a maxima in $\Delta P_{\mu \mu}$ and $\Delta P_{e \mu}$ for $n=2$, minima for $n=1$ and back again a maxima for $n=1$, as was expected from eqs. (3.3) and (3.4). This flipping of $\Delta P_{\mu \mu}$ with neutrino energy is also a signal for the octant of $\theta_{12}$. The presence of $\Delta m_{21}^{2}$ has two-fold effect. Firstly, it dampens $\Delta P$ as we go to higher energies. This comes from the first oscillatory term $\sin \left(\Delta m_{21}^{2} L / 4 E\right)$ in eqs. (3.3) and (3.4). Since this term decreases as we increase 


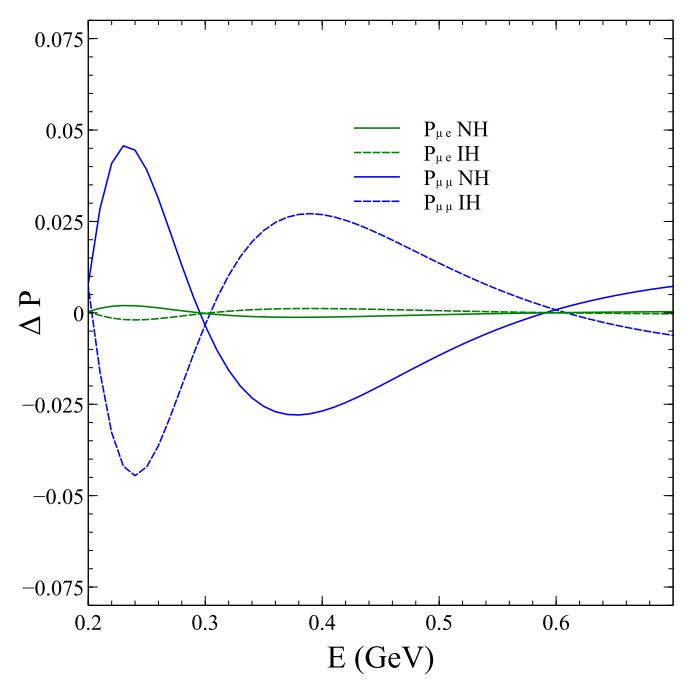

Figure 1. Difference in probability as a function of energy. The plot is shown for $L=295 \mathrm{~km}$ and without matter effect. The black lines show $\Delta P_{\mu \mu}$ while the green lines show $\Delta P_{\mu e}$. The solid lines are for $\mathrm{NH}$ while the dashed lines are for $\mathrm{IH}$.

energy, the damping is seen to increase with energy in figure 1 . Secondly, it determines the exact position of the $\Delta P$ extrema according to the second oscillatory term.

A comparison of eqs. (3.3) and (3.4) shows that $\Delta P_{\mu e} / \Delta P_{\mu \mu}=s_{13}^{2} /\left(\cos 2 \theta_{23}+s_{23}^{2} c_{13}^{2}\right)$, which for maximal $\theta_{23}$ gives $\Delta P_{\mu e} / \Delta P_{\mu \mu}=2 s_{13}^{2} / c_{13}^{2}$. Thus, for the measured value of $\theta_{13}$, $\Delta P_{\mu e}$ is suppressed by two orders of magnitude compared to $\Delta P_{\mu \mu}$. This difference can be seen in figure 1 and hence we conclude that in long-baseline experiments, it is the $P_{\mu \mu}$ (disappearance) channel which will bring in the sensitivity to the octant of $\theta_{12}$, while the role of the $P_{\mu e}$ (appearance) channel will be seen to be minimal.

Let us next look at the $\bar{\nu}_{e}$ survival probability that is relevant for the reactor experiments,

$$
P_{e e}=1-\sin ^{2} 2 \theta_{12} c_{13}^{4} \sin ^{2} \frac{\Delta m_{21}^{2} L}{4 E}-\sin ^{2} 2 \theta_{13}\left[c_{12}^{2} \sin ^{2} \frac{\Delta m_{31}^{2} L}{4 E}+s_{12}^{2} \sin ^{2} \frac{\Delta m_{32}^{2} L}{4 E}\right] .
$$

The second term in this expression is the leading term in the probability driven by $\Delta m_{21}^{2}$ oscillations and depends on the solar mixing angle as $\sin ^{2} 2 \theta_{12}$. This is the term that gives an experiment like JUNO its sensitivity to $\sin ^{2} 2 \theta_{12}$. However, since this term depends on $\sin ^{2} 2 \theta_{12}$ and not on $\sin ^{2} \theta_{12}$ (or $\cos ^{2} \theta_{12}$ ), it does not bring any sensitivity to the octant of $\theta_{12}$. The sensitivity to the octant of $\theta_{12}$ comes from the sub-dominant third term. This is the interference term between the solar and atmospheric frequencies. It comes from the full three-generation probability and indeed is the term that also brings the hierarchy sensitivity in JUNO. The similarity between this term and the third and fourth terms in eq. (3.1) is uncanny. Just as in the case of $P_{\mu \mu}$, this term depends on the octant of $\theta_{12}$ as long as one can distinguish between the oscillations driven by $\Delta m_{31}^{2}$ and $\Delta m_{32}^{2}$. The difference in $P_{e e}$ between $\sin ^{2} \theta_{12}$ and $\sin ^{2} \theta_{12}^{D}=\cos ^{2} \theta_{12}$ is given as

$$
\Delta P_{e e}=\sin ^{2} 2 \theta_{13} \cos 2 \theta_{12} \sin \left(\frac{\Delta m_{21}^{2} L}{4 E}\right) \sin \left(\frac{\left(2 \Delta m_{31}^{2}-\Delta m_{21}^{2}\right) L}{4 E}\right) .
$$


Comparison of eqs. (3.3), (3.4) and (3.6) shows that the oscillatory part in the respective $\Delta P_{\alpha \beta}$ are the same. The only difference is in the part which contain the mixing angles. We see that

$$
\frac{\Delta P_{e e}}{\Delta P_{\mu \mu}}=\frac{s_{13}^{2}}{s_{23}^{2}\left(c_{23}^{2}-s_{23}^{2} s_{13}^{2}\right)},
$$

which for maximal $\theta_{23}$ mixing reduces to

$$
\frac{\Delta P_{e e}}{\Delta P_{\mu \mu}}=4 \frac{s_{13}^{2}}{c_{13}^{2}}
$$

Hence, we see that the $\theta_{12}$ octant sensitivity is suppressed in the $P_{e e}$ channel compared to the $P_{\mu \mu}$ channel by a factor of $4 \tan ^{2} \theta_{13}$. For the sake of completeness, we also note that

$$
\frac{\Delta P_{e e}}{\Delta P_{\mu e}}=\frac{1}{s_{23}^{2}}
$$

which means that the octant sensitivity in the $P_{e e}$ channel is about twice as much as that in the $P_{\mu e}$ channel. Hence, from the discussion with the probabilities we see that the $\theta_{12}$ octant sensitivity appears to be maximum for $P_{\mu \mu}$ and minimum for $P_{\mu e}$, with the $P_{e e}$ channel being significantly less sensitive to $\theta_{12}$ octant compared to $P_{\mu \mu}$, but a factor of two more sensitive compared to the $P_{\mu e}$ channel. Nonetheless, as we will see in the next section, the contribution from the reactor antineutrino experiments is crucial for determining the octant of $\theta_{12}$ and without combining information from both $P_{\mu \mu}$ and $P_{e e}$ channels it will be impossible to determine the octant of $\theta_{12}$.

\section{Mass squared difference and $\theta_{12}$}

We begin by showing the sensitivity of the long-baseline experiments and then add the information from reactor experiment JUNO to study the sensitivity of oscillation experiments to octant of $\theta_{12}$. We show in figure 2 the $\chi^{2}$ and hence the significance with which T2HK will be able to distinguish LMA from DLMA, if all oscillation parameters are kept fixed at their assumed true value in the fit. We have taken the experimental settings for T2HK as given in [25] and considered the mock data corresponding to running the experiment for $2.5+7.5$ years in $\nu+\bar{\nu}$ mode, respectively. The mock data is generated at $\sin ^{2} \theta_{12}=0.31$ and we want to rule out the higher octant of $\theta_{12}$ in general and the DLMA solution in particular. Shown are the $\chi^{2}$ obtained from the analysis of disappearance data, appearance data, and both data sets combined. The appearance channel on its own is unable to resolve the degeneracy. However, it puts a weak lower and upper limit on $\sin ^{2} \theta_{12}$, as $P_{\mu e}$ depends on $\sin ^{2} 2 \theta_{12}$. We see that the disappearance data alone can rule out the DLMA solution at more than $5 \sigma$, if all the other parameters were known.

However, the other oscillation parameters are not completely known, and hence should be allowed to vary to account for correlations between them. Indeed there is a correlation between $\Delta m_{31}^{2}$ and $\theta_{12}$, which completely spoils this sensitivity and which we discuss next. Instead of keeping the oscillation parameters fixed as in figure 2, we now allow them to vary 


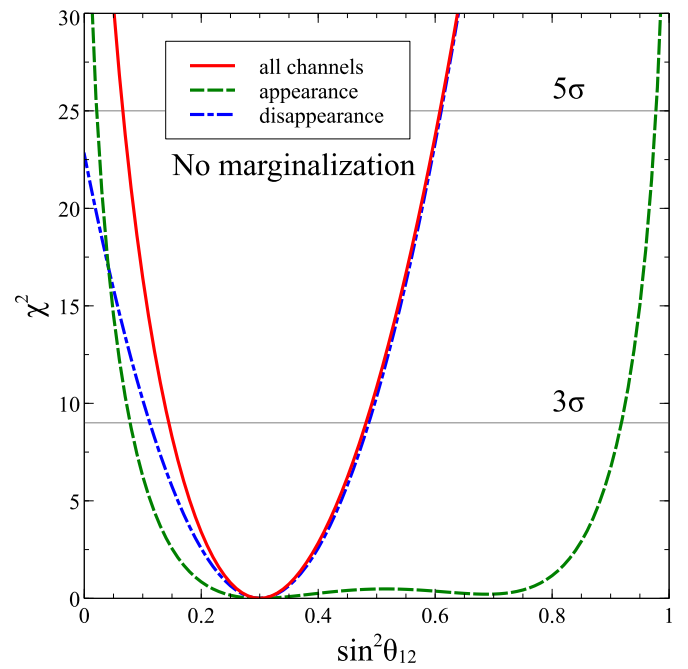

Figure 2. $\chi^{2}$ as a function of $\sin ^{2} \theta_{12}$ for T2HK mock data generated at $\sin ^{2} \theta_{12}=0.31$ corresponding to the LMA solution and fitted by varying $\sin ^{2} \theta_{12}$ while all other parameters are fixed. Results are shown for analysis of appearance channel data only, disappearance channel data only and for the combination of both appearance and disappearance channel data.

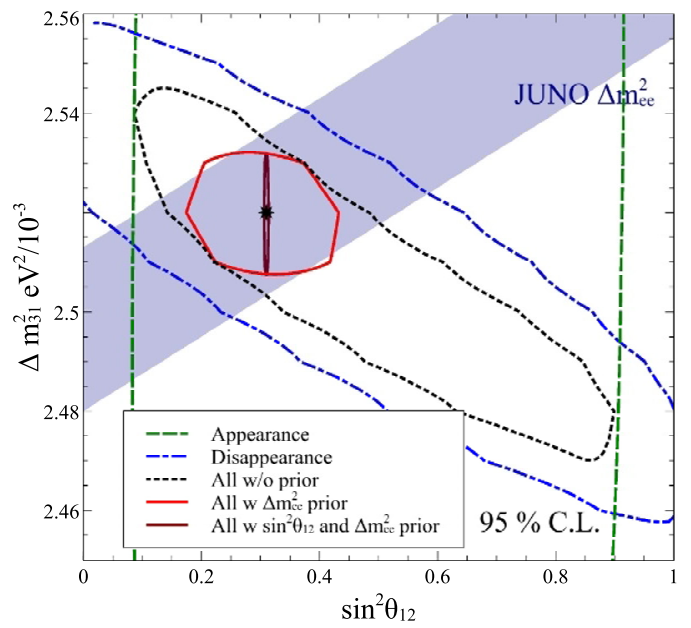

Figure 3. The $95 \%$ C.L. expected allowed areas in the $\Delta m_{31}^{2}-\sin ^{2} \theta_{12}$ plane for T2HK. The blue dot-dashed contour is obtained using T2HK disappearance channel only, the green dashed curve using T2HK appearance channel only and the black dotted curve using both. The blue shaded region is the JUNO prior on $\Delta m_{e e}^{2}$. The red solid contour is obtained when we apply the prior from JUNO on $\Delta m_{e e}^{2}$ and the dark red contour is obtained when we also add the $\sin ^{2} 2 \theta_{12}$ prior along with the prior on $\Delta m_{e e}^{2}$.

as detailed in table 1. Figure 3 presents the $95 \%$ C.L. allowed area in the $\Delta m_{31}^{2}-\sin ^{2} \theta_{12}$ plane expected at T2HK from disappearance channel (blue dot-dashed curve), appearance channel (green dashed curve) and by combining the two (black dotted curve). The contours are marginalised over $\theta_{13}$ and $\delta_{\mathrm{CP}}$. The assumed true value in this parameter space is shown by the black star. We find that once we allow $\Delta m_{31}^{2}$ to vary, the constraint on $\sin ^{2} \theta_{12}$ is seen to be completely lost. The appearance channel puts only a weak constraint on 
$\sin ^{2} 2 \theta_{12}$, as was the case in figure 2. However, for the disappearance channel the effect is drastic and we find that nearly the entire $\sin ^{2} \theta_{12}$ range gets allowed. This happens because of the anti-correlation between $\Delta m_{31}^{2}$ and $\sin ^{2} \theta_{12}$ in the $\nu_{\mu}$ survival probability which can be explained easily if we rewrite eq. (3.1) in terms of an effective mass squared difference $\Delta m_{\mu \mu}^{2}$ such that [31-33]

$$
\begin{aligned}
P_{\mu \mu} & \simeq 1-4 \sin ^{2} \theta_{23} \cos ^{2} \theta_{13}\left(1-\sin ^{2} \theta_{23} \cos ^{2} \theta_{13}\right) \sin ^{2}\left(\frac{\Delta m_{\mu \mu}^{2} L}{4 E}\right), \\
\Delta m_{\mu \mu}^{2} & =\Delta m_{31}^{2} \Delta m_{21}^{2}\left(\cos ^{2} \theta_{12}-\cos \delta \sin \theta_{13} \sin 2 \theta_{12} \tan \theta_{23}\right)
\end{aligned}
$$

The long-baseline experiments measure the effective parameter $\Delta m_{\mu \mu}^{2}$ which depends on $\Delta m_{31}^{2}$ and $\sin ^{2} \theta_{12}$ in an anti-correlated way and hence $P_{\mu \mu}$ alone will never be able to measure $\sin ^{2} \theta_{12}$ in a long-baseline experiment. A given measured value of $\Delta m_{\mu \mu}^{2}$ can always be reproduced for lower $\Delta m_{31}^{2}$ and higher $\sin ^{2} \theta_{12}$, and/or vice-versa. Adding the $P_{\mu e}$ channel to the analysis along with the $P_{\mu \mu}$ channel (shown by black short-dashed line) improves the constraint on $\sin ^{2} \theta_{12}$ only marginally on the two edges of the scale, as was expected from the green-dashed lines in figures 2. However, this is unable to break the $\Delta m_{31}^{2}$ and $\sin ^{2} \theta_{12}$ anti-correlation coming from $\Delta m_{\mu \mu}^{2}$ and as a result the DLMA solution continues to get allowed. In order to remedy this, we need an independent measurement of $\Delta m_{31}^{2}$ which can break the $\Delta m_{31}^{2}$ and $\sin ^{2} \theta_{12}$ anti-correlation in the $P_{\mu \mu}$ channel.

Such an independent channel can be the $P_{e e}$ channel, as discussed before. As in the case of $\nu_{\mu}$ disappearance, we can write the $\bar{\nu}_{e}$ disappearance probability in terms of an effective mass squared difference $\Delta m_{e e}^{2}[12]$ as

$$
\begin{aligned}
P_{e e}= & 1-\sin ^{2} 2 \theta_{13}\left[1-\sqrt{1-\sin ^{2} 2 \theta_{12} \sin ^{2} \Delta_{21}} \cos \left(2 \Delta m_{e e}^{2} \pm \phi\right)\right] \\
& -\cos ^{4} \theta_{13} \sin ^{2} 2 \theta_{12} \sin ^{2} \Delta_{21}
\end{aligned}
$$

where $\Delta m_{e e}^{2}$ and $\phi$ carry the $\theta_{12}$ octant dependence of $P_{e e}$ and are given by

$$
\begin{aligned}
\Delta m_{e e}^{2} & =\cos ^{2} \theta_{12} \Delta m_{31}^{2}+\sin ^{2} \theta_{12} \Delta m_{32}^{2}, \\
\sin \phi & =\frac{c_{12}^{2} \sin \left(2 s_{12}^{2} \Delta_{21}\right)-s_{12}^{2} \sin \left(2 c_{12}^{2} \Delta_{21}\right)}{\sqrt{1-\sin ^{2} 2 \theta_{12} \sin ^{2} \Delta_{21}}} .
\end{aligned}
$$

We have used $\Delta_{21}=\Delta m_{21}^{2} L / 4 E$ in the above as shorthand and \pm in eq. (4.2) corresponds to the neutrino mass hiearchy, + for normal and - for inverted. From eqs. (4.3) and (4.4) it is clear that $\phi$ is smaller than $\Delta m_{e e}^{2}$. Note that the dependence of $\Delta m_{e e}^{2}$ on $\theta_{12}$ is complementary to what we had for $\Delta m_{\mu \mu}^{2}$. While the former depends on $\sin ^{2} \theta_{12}$, the latter on $\cos ^{2} \theta_{12}$. We use here the expected constraints from the JUNO [12] experiment which will observe $\bar{\nu}_{e}$ disappearance probability $P_{e e}$ and will measure the effective mass squared difference $\Delta m_{e e}^{2}$ and $\sin ^{2} 2 \theta_{12}$ to a very high precision. We show in the blue band the $95 \%$ C.L. expected allowed area in the $\Delta m_{31}^{2}-\sin ^{2} \theta_{12}$ plane from JUNO taken from [12]. Since $\Delta m_{31}^{2}$ and $\sin ^{2} \theta_{12}$ are correlated for $\Delta m_{e e}^{2}$ measured in JUNO, we get a slanting bar in this plane, corresponding to this constraint. This band intersects with the band/contour 

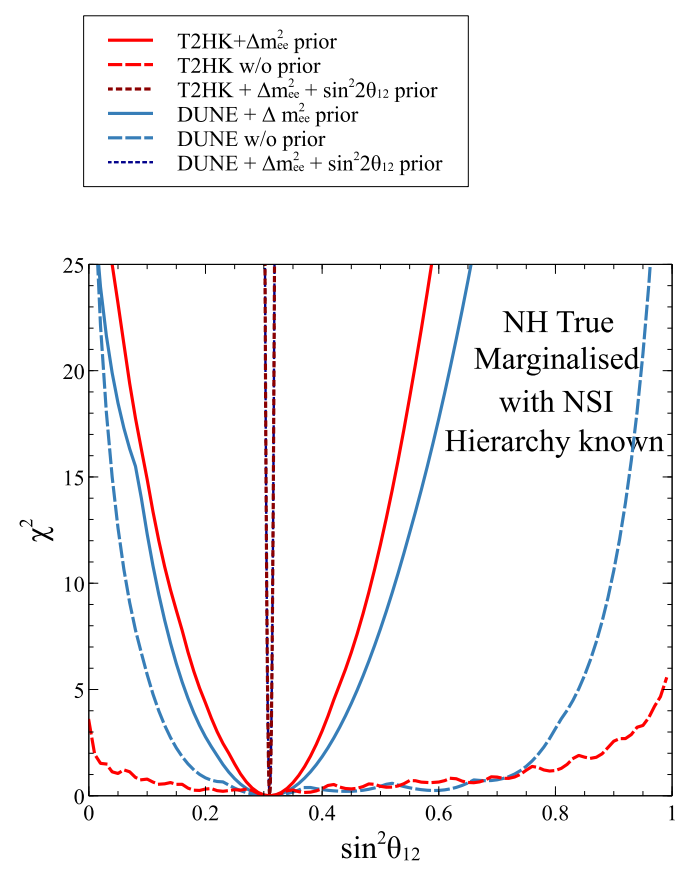

Figure 4. $\chi^{2}$ as a function of $\sin ^{2} \theta_{12}$ for mock data generated $\operatorname{at}^{2} \sin _{12}=0.31$. The parameters $\delta_{\mathrm{CP}},\left|\Delta m_{31}^{2}\right|$ and $\epsilon_{e e}$ are allowed to vary freely in the fit. Shown are the sensitivity expected at T2HK as well as DUNE with and without prior from JUNO.

corresponding to T2HK around the best-fit point (star). This happens because $\Delta m_{31}^{2}$ and $\sin ^{2} \theta_{12}$ are correlated for $\Delta m_{e e}^{2}$, while they are anti-correlated for $\Delta m_{\mu \mu}^{2}$, making the two bands slant in opposite directions. Including the JUNO constraint on $\Delta m_{e e}^{2}$ as a prior in our analysis of the T2HK data gives us the contour shown by the red solid curve. The degeneracy between $\Delta m_{31}^{2}$ and $\sin ^{2} \theta_{12}$ is broken, giving us a unique solution in $\sin ^{2} \theta_{12}$ and the DLMA solution can now be completely ruled out. If we also add the expected JUNO constraint on $\theta_{12}$, we get the sharp narrow contour shown by the maroon solid line.

As mentioned above, in addition to $\Delta m_{e e}^{2}, P_{e e}$ also depends on the octant of $\theta_{12}$ via the $\phi$ terms. While this term is sub-dominant, it brings a difference in the $P_{e e}$ channel between the two octants of $\theta_{12}$. This $\phi$ dependence in $P_{e e}$ can be seen only in an experiment like JUNO which has very good energy resolution. This could enable JUNO alone to measure the octant of $\theta_{12}$ if the neutrino mass hierarchy was already known, as was pointed out in [8].

\section{Comparing T2HK with DUNE}

In figure 4 we show the $\chi^{2}$ as a function of $\sin ^{2} \theta_{12}$ for mock data generated at $\sin ^{2} \theta_{12}=$ 0.31 . We have shown here the expected $\chi^{2}$ from combined appearance and disappearance data at T2HK as well as DUNE. The oscillation parameters $\delta_{\mathrm{CP}}$ as well as $\left|\Delta m_{31}^{2}\right|$ are varied freely in the fit. We have also varied $\epsilon_{e e}$ in the fit. Results are shown without taking any prior from JUNO (dashed lines), when the JUNO prior on $\Delta m_{e e}^{2}$ is included (solid lines) and when the JUNO prior on $\sin ^{2} 2 \theta_{12}$ is also added along with the $\Delta m_{e e}^{2}$ prior (dotted 

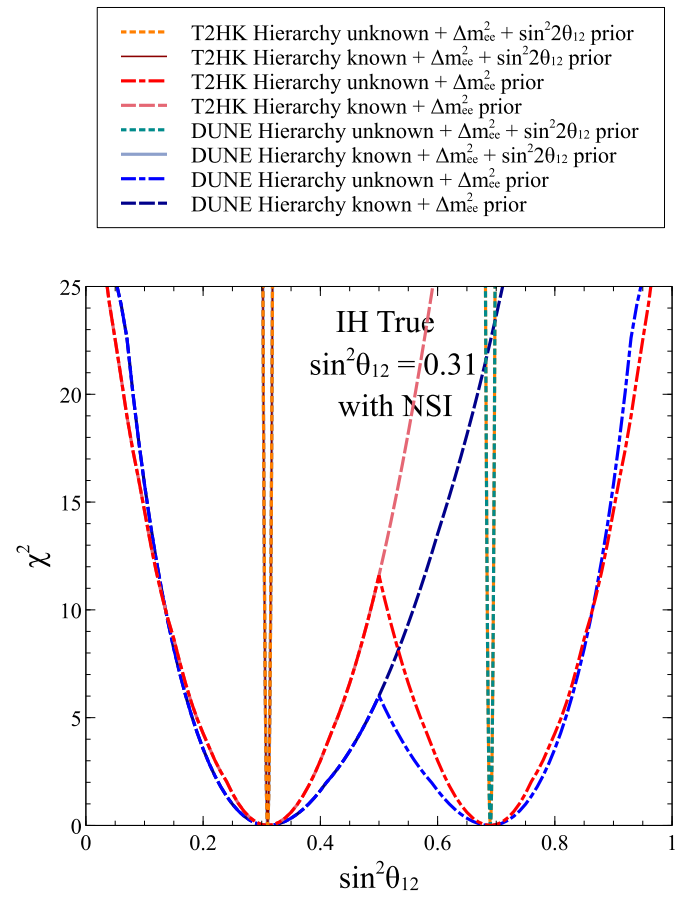

Figure 5. The impact of the neutrino mass hierarchy on the determination of the octant of $\theta_{12}$.

lines). As in figure 3, we see that without the prior on $\Delta m_{e e}^{2}$ from JUNO, it is impossible for the long-baseline experiments to put any meaningful constraint on $\sin ^{2} \theta_{12}$. However, once the information on $\Delta m_{e e}^{2}$ from JUNO is included, the disappearance channel at these experiments can rule out the DLMA solution at more than $5 \sigma$ significance at T2HK and more than $4 \sigma$ at DUNE. We also see that when the $\sin ^{2} 2 \theta_{12}$ prior is added from JUNO, the precision of $\theta_{12}$ is strictly determined by the JUNO prior.

\section{Role of neutrino mass hierarchy}

So long we had fixed the neutrino mass hierarchy, assuming that it will be determined by a non-oscillation experiment. Neutrino-less double beta decay could fix the neutrino mass hierarchy $[34,35]$. Information on neutrino mass hierarchy could also come from future cosmological data $[23,36]$. We have already stressed the caveats associated with this assumption in the introduction. Here we lift this assumption and study the phenomenological implications of what happens if hierarchy is unknown.

In figure 5, we show the impact of mass hierarchy on ruling out the DLMA solution. We present four cases each for T2HK and DUNE. The solid lines are for the cases when we take hierarchy to be known and add both $\Delta m_{e e}^{2}$ and $\sin ^{2} 2 \theta_{12}$ priors from JUNO, the dotted lines are for the cases when both priors are added but the hierarchy is assumed to be unknown, the dashed lines are for only $\Delta m_{e e}^{2}$ prior and hierarchy unknown and the dashed-dotted lines are with $\Delta m_{e e}^{2}$ prior but hierarchy known. The data is generated using the parameters given in section 2 and mass hierarchy is assumed to be inverted in data. For the cases where the mass hierarchy is taken as known, we keep the mass hierarchy 
fixed in our fit, while for cases when it is taken as unknown we marginalise our $\chi^{2}$ over mass hierarchy. We take no NSI in the data while in the fit, we varied the oscillation parameters as well as NSI parameters in the ranges gives in table 1. We see that due to the generalised mass hierarchy degeneracy, for all the hierarchy unknown cases, the fit in the presence of NSI parameters gives two degenerate solutions corresponding to the LMA (true solution) and DLMA (fake solution). On the other hand, if we know the mass hierarchy through measurements at neutrino-less double beta decay experiments and/or cosmology, the corresponding $\chi^{2}$ show that the DLMA solution can be disfavoured at a very high significance once prior on $\Delta m_{e e}^{2}$ is applied along with the long-baseline data. We note that the entire higher octant corresponding to $\sin ^{2} \theta_{12}>0.5$ can be ruled out at greater than $3 \sigma$ by data from T2HK. For DUNE the significance is slightly less, but still competitive. As we saw in figure 4 , the role of the prior on $\sin ^{2} 2 \theta_{12}$ is to increase the precision on the solar mixing angle.

\section{Conclusions}

In presence of NSIs the solar neutrino data is known to give two (nearly) degenerate solutions with same $\Delta m_{21}^{2}$ but with $\sin ^{2} \theta_{12} \simeq 0.3$ and $\sin ^{2} \theta_{12}^{D} \simeq 0.7$, known as the LMA and DLMA solutions, respectively. The existence of this degeneracy has both model building as well as phenomenological implications. One being the so-called generalised mass hierarchy degeneracy wherein the neutrino mass matrix in vacuum remains invariant under the simultaneous exchange of $\Delta m_{31}^{2} \leftrightarrow-\Delta m_{32}^{2}, \sin ^{2} \theta_{12} \leftrightarrow \cos ^{2} \theta_{12}$ and $\delta_{c p} \leftrightarrow \pi-\delta_{c p}$. This is a problem for experiments like JUNO which aim to measure the neutrino mass hierarchy by observing neutrino oscillations in vacuum. While presence of matter effect could resolve this degeneracy for standard neutrinos, in presence of NSI the transformations $\epsilon_{e e} \rightarrow \epsilon_{e e}-2, \epsilon_{e \mu} \rightarrow \epsilon_{e \mu}^{*}, \epsilon_{e \tau} \rightarrow \epsilon_{e \tau}^{*}$ and all other $\epsilon_{\alpha \beta} \rightarrow-\epsilon_{\alpha \beta}^{*}$ are allowed and hence the generalised mass hierarchy degeneracy is restored, making simultaneous measurement of neutrino mass hierarchy and octant of $\theta_{12}$ impossible at neutrino oscillation experiments alone.

While this is well known in the literature, in this paper we showed that even if the neutrino mass hierarchy was measured in a non-oscillation such as neutrino-less double beta decay, long-baseline experiments alone would still not be able to determine the octant of $\theta_{12}$. This happens because the dependence on octant of $\theta_{12}$ in these experiments come from the measurement of the effective atmospheric mass squared difference. For given measured value of $\Delta m_{\mu \mu}^{2}$, oscillation parameters $\Delta m_{31}^{2}$ and $\sin ^{2} \theta_{12}$ are anticorrelated to each other. This intrinsic anticorrelation makes it impossible to measure the octant of $\theta_{12}$ using the $P_{\mu \mu}$ channel alone. The $P_{e e}$ channel has $\Delta m_{31}^{2}$ and $\sin ^{2} \theta_{12}$ correlated and hence, combining the reactor data with the long-baseline data cancels the mentioned (anti)correlation and gives us a unique solution for the octant of $\sin ^{2} \theta_{12}$. This can therefore resolve the LMA vs DLMA conundrum.

To summarise,

- Due to the generalised mass hierarchy degeneracy, one cannot measure $\theta_{12}$ and mass hierarchy simultaneously in any oscillation experiment. This is well known in the literature. 
- In this paper we showed that even if the mass hierarchy was known, it would still not be possible to determine the octant of $\theta_{12}$ in a long-baseline experiment.

- We showed that the dependence of the probability on the octant of $\theta_{12}$ came via the effective atmospheric mass squared difference that is $\Delta m_{\mu \mu}^{2}$ for long baseline experiments. Since $\Delta m_{\mu \mu}^{2}=\Delta m_{31}^{2}-\Delta m_{21}^{2}\left(\cos ^{2} \theta_{12}-\cos \delta \sin \theta_{13} \sin 2 \theta_{12} \tan \theta_{23}\right)$, this gives a anticorrelation between $\Delta m_{31}^{2}$ and $\sin ^{2} \theta_{12}$, and hence, no matter how precisely the effective mass squared difference is measured, we get a whole band of allowed values of $\sin ^{2} \theta_{12}$.

- However, once JUNO and long-baseline experiment(s) are combined, the (anti)correlation between $\Delta m_{31}^{2}$ and $\sin ^{2} \theta_{12}$ gets cancelled breaking the $\theta_{12}$ octant degenracy.

\section{Acknowledgments}

We acknowledge support from HRI where major part of this work was completed. The HRI cluster computing facility (http://cluster.hri.res.in) is also acknowledged. We are indebted to T. Schwetz for very insightful discussions. We also thank S. Goswami for her valuable inputs and suggestions. This project has received funding from the European Union's Horizon 2020 research and innovation programme InvisiblesPlus RISE under the Marie Sklodowska-Curie grant agreement No 690575. This project has received funding from the European Union's Horizon 2020 research and innovation programme Elusives ITN under the Marie Sklodowska-Curie grant agreement No. 674896.

Open Access. This article is distributed under the terms of the Creative Commons Attribution License (CC-BY 4.0), which permits any use, distribution and reproduction in any medium, provided the original author(s) and source are credited.

\section{References}

[1] SNO collaboration, Electron energy spectra, fluxes, and day-night asymmetries of B-8 solar neutrinos from measurements with $\mathrm{NaCl}$ dissolved in the heavy-water detector at the Sudbury Neutrino Observatory, Phys. Rev. C 72 (2005) 055502 [nucl-ex/0502021] [INSPIRE].

[2] KAMLAND collaboration, Precision measurement of neutrino oscillation parameters with KamLAND, Phys. Rev. Lett. 100 (2008) 221803 [arXiv:0801.4589] [InSPIRE].

[3] I. Esteban, M.C. Gonzalez-Garcia, A. Hernandez-Cabezudo, M. Maltoni and T. Schwetz, Global analysis of three-flavour neutrino oscillations: synergies and tensions in the determination of $\theta_{23}, \delta_{\mathrm{CP}}$, and the mass ordering, JHEP 01 (2019) 106 [arXiv:1811.05487] [INSPIRE].

[4] O.G. Miranda, M.A. Tortola and J.W.F. Valle, Are solar neutrino oscillations robust?, JHEP 10 (2006) 008 [hep-ph/0406280] [INSPIRE].

[5] F.J. Escrihuela, O.G. Miranda, M.A. Tortola and J.W.F. Valle, Constraining nonstandard neutrino-quark interactions with solar, reactor and accelerator data, Phys. Rev. D 80 (2009) 105009 [Erratum ibid. 80 (2009) 129908] [arXiv:0907.2630] [INSPIRE]. 
[6] L. Wolfenstein, Neutrino oscillations in matter, Phys. Rev. D 17 (1978) 2369 [InSPIRE].

[7] M.C. Gonzalez-Garcia and M. Maltoni, Determination of matter potential from global analysis of neutrino oscillation data, JHEP 09 (2013) 152 [arXiv:1307.3092] [INSPIRE].

[8] P. Bakhti and Y. Farzan, Shedding light on LMA-dark solar neutrino solution by medium baseline reactor experiments: JUNO and RENO-50, JHEP 07 (2014) 064 [arXiv:1403.0744] [INSPIRE].

[9] P. Coloma and T. Schwetz, Generalized mass ordering degeneracy in neutrino oscillation experiments, Phys. Rev. D 94 (2016) 055005 [Erratum ibid. 95 (2017) 079903] [arXiv: 1604.05772] [INSPIRE].

[10] P. Coloma, P.B. Denton, M.C. Gonzalez-Garcia, M. Maltoni and T. Schwetz, Curtailing the dark side in non-standard neutrino interactions, JHEP 04 (2017) 116 [arXiv:1701.04828] [INSPIRE].

[11] I. Esteban, M.C. Gonzalez-Garcia, M. Maltoni, I. Martinez-Soler and J. Salvado, Updated constraints on non-standard interactions from global analysis of oscillation data, JHEP 08 (2018) 180 [arXiv: 1805.04530] [INSPIRE].

[12] JUNO collaboration, Neutrino physics with JUNO, J. Phys. G 43 (2016) 030401 [arXiv: 1507.05613] [INSPIRE].

[13] S. Choubey, S.T. Petcov and M. Piai, Precision neutrino oscillation physics with an intermediate baseline reactor neutrino experiment, Phys. Rev. D 68 (2003) 113006 [hep-ph/0306017] [INSPIRE].

[14] DUNE collaboration, The DUNE far detector interim design report volume 1: physics, technology and strategies, arXiv: 1807.10334 [INSPIRE].

[15] P. Coloma, M.C. Gonzalez-Garcia, M. Maltoni and T. Schwetz, COHERENT enlightenment of the neutrino dark side, Phys. Rev. D 96 (2017) 115007 [arXiv: 1708.02899] [InSPIRE].

[16] CHARM collaboration, Experimental verification of the universality of $\nu_{e}$ and $\nu_{\mu}$ coupling to the neutral weak current, Phys. Lett. B 180 (1986) 303 [INSPIRE].

[17] NuTEV collaboration, A precise determination of electroweak parameters in neutrino nucleon scattering, Phys. Rev. Lett. 88 (2002) 091802 [Erratum ibid. 90 (2003) 239902] [hep-ex/0110059] [INSPIRE].

[18] COHERENT collaboration, COHERENT collaboration data release from the first observation of coherent elastic neutrino-nucleus scattering, arXiv:1804.09459 [INSPIRE].

[19] P.B. Denton, Y. Farzan and I.M. Shoemaker, Testing large non-standard neutrino interactions with arbitrary mediator mass after COHERENT data, JHEP 07 (2018) 037 [arXiv: 1804.03660] [INSPIRE].

[20] P. Coloma, I. Esteban, M.C. Gonzalez-Garcia and M. Maltoni, Improved global fit to non-standard neutrino interactions using COHERENT energy and timing data, JHEP 02 (2020) 023 [arXiv: 1911.09109] [INSPIRE].

[21] K.N. Vishnudath, S. Choubey and S. Goswami, New sensitivity goal for neutrinoless double beta decay experiments, Phys. Rev. D 99 (2019) 095038 [arXiv:1901.04313] [INSPIRE].

[22] C. Giunti and E.M. Zavanin, Predictions for neutrinoless double-beta decay in the $3+1$ sterile neutrino scenario, JHEP 07 (2015) 171 [arXiv: 1505.00978] [INSPIRE]. 
[23] S. Roy Choudhury and S. Hannestad, Updated results on neutrino mass and mass hierarchy from cosmology with Planck 2018 likelihoods, JCAP 07 (2020) 037 [arXiv:1907.12598] [INSPIRE].

[24] DUNE collaboration, Long-Baseline Neutrino Facility (LBNF) and Deep Underground Neutrino Experiment (DUNE): conceptual design report, volume 2: the physics program for DUNE at LBNF, arXiv:1512.06148 [INSPIRE].

[25] Hyper-Kamiokande Proto- collaboration, Physics potential of a long-baseline neutrino oscillation experiment using a J-PARC neutrino beam and Hyper-Kamiokande, PTEP 2015 (2015) 053C02 [arXiv:1502.05199] [INSPIRE].

[26] T2K collaboration, The T2K experiment, Nucl. Instrum. Meth. A 659 (2011) 106 [arXiv: 1106.1238] [INSPIRE].

[27] Hyper-Kamiokande collaboration, Physics potentials with the second Hyper-Kamiokande detector in Korea, PTEP 2018 (2018) 063C01 [arXiv:1611.06118] [INSPIRE].

[28] P. Huber, M. Lindner and W. Winter, Simulation of long-baseline neutrino oscillation experiments with GLoBES (General Long Baseline Experiment Simulator), Comput. Phys. Commun. 167 (2005) 195 [hep-ph/0407333] [INSPIRE].

[29] P. Huber, J. Kopp, M. Lindner, M. Rolinec and W. Winter, New features in the simulation of neutrino oscillation experiments with GLoBES 3.0: General Long Baseline Experiment Simulator, Comput. Phys. Commun. 177 (2007) 432 [hep-ph/0701187] [INSPIRE].

[30] M. Blennow and E. Fernandez-Martinez, Neutrino oscillation parameter sampling with MonteCUBES, Comput. Phys. Commun. 181 (2010) 227 [arXiv:0903.3985] [INSPIRE].

[31] A. de Gouvêa, J. Jenkins and B. Kayser, Neutrino mass hierarchy, vacuum oscillations, and vanishing $|U(e 3)|$, Phys. Rev. D 71 (2005) 113009 [hep-ph/0503079] [INSPIRE].

[32] H. Minakata, H. Nunokawa, S.J. Parke and R. Zukanovich Funchal, Determining neutrino mass hierarchy by precision measurements in electron and muon neutrino disappearance experiments, Phys. Rev. D 74 (2006) 053008 [hep-ph/0607284] [InSPIRE].

[33] L. Zhan, Y. Wang, J. Cao and L. Wen, Determination of the Neutrino Mass Hierarchy at an Intermediate Baseline, Phys. Rev. D 78 (2008) 111103 [arXiv:0807.3203] [InSPIRE].

[34] W.H. Furry, On transition probabilities in double beta-disintegration, Phys. Rev. 56 (1939) 1184 [INSPIRE].

[35] S. Dell'Oro, S. Marcocci, M. Viel and F. Vissani, Neutrinoless double beta decay: 2015 review, Adv. High Energy Phys. 2016 (2016) 2162659 [arXiv:1601.07512] [INSPIRE].

[36] S. Vagnozzi et al., Unveiling $\nu$ secrets with cosmological data: neutrino masses and mass hierarchy, Phys. Rev. D 96 (2017) 123503 [arXiv:1701.08172] [INSPIRE]. 